\title{
Reduced Common Molecular Orbital Basis for Nonorthogonal Configuration Interaction
}

\author{
R. K. Kathir, Coen de Graaf,* Ria Broer, and Remco W. A. Havenith* \\ Cite This: J. Chem. Theory Comput. 2020, 16, 2941-2951 \\ Read Online
}

ABSTRACT: Electron and charge transfers are part of many vital processes in nature and technology. Ab initio descriptions of these processes provide useful insights that can be utilized for applications. A combination of the embedded cluster material model and nonorthogonal configuration interaction (NOCI), in which the cluster wave functions are expanded in many-electron basis functions (MEBFs) consisting of spin-adapted, antisymmetrized products of multiconfigurational wave functions of fragments (which are usually molecules) in the cluster, appears to provide a compromise between accuracy and calculation time. Additional advantages of this NOCI-Fragments approach are the chemically

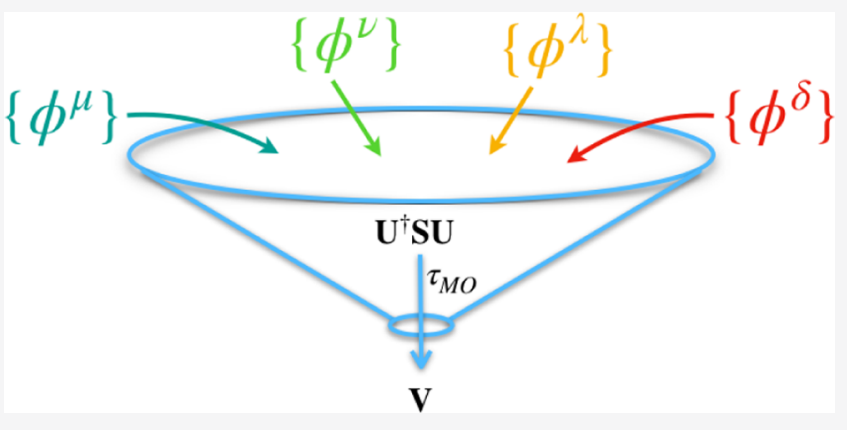
convenient interpretation of the wave function in terms of molecular states, and the direct accessibility of electronic coupling between diabatic states to describe energy and electron transfer processes. Bottlenecks in this method are the large number of two-electron integrals that have to be handled for the calculation of an electronic coupling matrix element and the enormous number of matrix elements over determinant pairs that have to be evaluated for the calculation of one matrix element between the MEBFs. We show here how we created a reduced common molecular orbital basis that is utilized to significantly reduce the number of two-electron integrals that need to be handled. The results obtained with this basis do not show any loss of accuracy in relevant quantities like electronic couplings and vertical excitation energies. We also show a significant reduction in computation time without loss in accuracy when matrix elements over determinant pairs with small weights are neglected in the NOCI. These improvements in the methodology render NOCI-Fragments to be also applicable to treat clusters of larger molecular systems with larger atomic basis sets and larger active spaces, as the computation time becomes dependent on the number of occupied orbitals and less dependent on the size of the active space.

\section{INTRODUCTION}

A detailed description of inter- and intramolecular transport of electrons or energy in the form of electronically excited states is essential to understand many processes in nature and in technological applications. ${ }^{1-4}$ For a theoretical understanding of the electronic structure of the molecules involved in these processes, one can opt for ab initio methods using either density functional theory (DFT) $)^{5,6}$ or wave function-based approaches. Often, such calculations are performed within the molecular orbital (MO) approximation, and DFT is typically accurate and computationally cheap enough to cover most applications. However, when high accuracy is required or the electronic structure cannot be reasonably described with a single electronic configuration, wave function-based approaches like coupled cluster $^{7-9}$ and multiconfigurational self-consistent field (SCF) ${ }^{10-12}$ come into play. Moreover, it is not straightforward to calculate matrix elements between diabatic states with DFT.

The use of one set of orthogonal one-electron functions (orbitals) to construct all Slater determinants in which the many-electron wave functions are expanded simplifies tremendously the computational procedure and is nowadays the dominant choice for wave function-based calculations. Alternatively, electronic structure calculations can also be performed within the valence bond (VB) approach, ${ }^{13}$ a computationally more complex, but chemically more intuitive method that is based on nonorthogonal orbitals. The VB description of chemical bonding remains very close to the concepts derived from the Lewis structures of molecules and is therefore very attractive to translate computational results into simple, chemically relevant concepts. A drawback is that accurate VB calculations are, in many cases, not feasible or extremely costly. Our NOCI approach is aimed at combining conceptual attractiveness with accuracy and computational feasibility.

Received: November 18, 2019

Published: April 11, 2020 
Coming back to the charge and excitation transfer processes mentioned before, significant changes can be expected when comparing the electronic structures of initial and final states of charge and excitation transfer processes such as

$$
\mathrm{A}+\mathrm{B} \rightarrow \mathrm{A}^{+}+\mathrm{B}^{-}
$$

and

$$
\mathrm{A}^{*}+\mathrm{B} \rightarrow \mathrm{A}+\mathrm{B}^{*}
$$

with A and B representing the ground-state electron distributions of two different molecules (or two fragments of the same molecule), $\mathrm{A}^{+}$and $\mathrm{B}^{-}$are the cationic and anionic states of $\mathrm{A}$ and $\mathrm{B}$, and $\mathrm{A}^{*}$ and $\mathrm{B}^{*}$ denote excited states localized at the fragments or molecules. Not only do the initial and final states differ in their leading electronic configuration but also in the shape of the optimal orbitals that describe the electron distributions. ${ }^{14} \mathrm{~A}$ multiconfigurational approach can certainly take into account the change in the leading electronic configuration, but the restriction of one set of orthogonal orbitals to describe both initial and final states makes it difficult to find an unbiased orbital description. Optimizing the orbitals for the $A-B$ state may result in a poor description of the $A^{+}-$ $\mathrm{B}^{-}$charge transfer state and similar $\mathrm{A}^{*}-\mathrm{B}$ optimized orbitals are not very well suited to describe the $A-B^{*}$ exciton-transfer state. The alternative, and more commonly used, choice of a state-average optimization of the orbitals is also not free of problems. Normally, the average cannot be restricted to the two states of interest only. The excited state is typically not the lowest excited state and has other electronic states close in energy, implying that the state-average procedure necessarily includes more states than strictly required.

In this study, we aim to combine the advantages of VB and MO theories and apply a strategy denoted NOCI-Fragments that, to some extent, can be characterized as a divide-andconquer scheme. Instead of directly heading for accurate approximations of the many-electron states of the A-B system, we separately calculate wave functions of the $A$ and $B$ fragments applying one of the standard (orthogonal) MObased approaches. Following the two examples from above, this implies six separate calculations: two to determine the wave functions of the ground states of $\mathrm{A}$ and $\mathrm{B}$; then the ionic states $\mathrm{A}^{+}$and $\mathrm{B}^{-}$; and, finally, the excited states $\mathrm{A}^{*}$ and $\mathrm{B}^{*}$. Each of these fragment wave functions is expressed in their own optimal set of orthogonal orbitals and, therefore, the orbitals of different sets may have nonzero mutual overlap. Next, we construct wave functions for the full system by making spin-adapted linear combinations of antisymmetrized product wave functions from the fragment wave functions. These so-called many-electron basis functions (MEBFs) are then used to build a configuration interaction (CI) matrix, which allows us to calculate the coupling between the diabatic states and, upon diagonalization of this nonorthogonal CI (NOCI) matrix, also to obtain the $\mathrm{N}$-electron wave functions and energies of the electronic states of the full system. Note that when the fragment wave functions used to build the MEBFs (or diabatic states) are calculated through a CASSCF procedure, there is no need to have the same active space size for the different wave functions of a fragment.

Computational approaches with nonorthogonal orbitals have recently generated renewed interest among several groups. Olsen and co-workers have developed an efficient implementation of multiconfigurational SCF in terms of nonorthogonal orbitals describing both an efficient algorithm for the CI and the orbital optimization steps. ${ }^{15}$ This was later extended by the implementation of a perturbative treatment of the dynamic correlation $^{16,17}$ and an orbital localization scheme. ${ }^{18}$ A second important recent contribution to the field was made by the group of Head-Gordon, who developed a spin-flip variant of nonorthogonal $\mathrm{CI}^{19}$ and a scheme based on Generalized Hartree-Fock reference wave functions, ${ }^{20}$ followed by the extension to $\mathrm{MP}^{21,22}$ and core-hole excitations. ${ }^{23,24}$ Here, we also mention the important contributions to the nonorthogonal approaches recently made within the framework of the valence bond theory of $\mathrm{Wu}$ et al., giving an important boost to the efficiency of VB calculations. ${ }^{25-28}$ Other recent contributions to the field were reported by Burton, Gross, and Thom, ${ }^{29,30}$ and Nite and Jiménez-Hoyos. ${ }^{31}$ They present nonorthogonal CI schemes to calculate multiconfigurational excited states using resonating Hartree-Fock ${ }^{32}$ or holomorphic Hartree-Fock ${ }^{33}$ reference wave functions.

Our implementation of NOCI has two important advantages in comparison to standard orthogonal MO approaches. First, all of the many-electron states are described in an unbiased way; each state is expressed in its own optimal orbitals. Second, the final wave function expansion of the electronic states remains very short, with typically less than $10 \mathrm{MEBFs}$, which by themselves are neat representations of easily identifiable electronic configurations. Hence, the interpretation of the final wave function is rather simple and stays close to the intuitive language of the VB theory.

Obviously, lifting the restriction of orthogonal orbitals comes at a cost. The calculation of two-electron matrix elements between multiconfigurational wave functions expressed in one and the same set of orthogonal orbitals is relatively easy. The Slater-Condon rules state that only determinant pairs with at most two differences in the orbital occupancy have a nonzero contribution to a matrix element of two-electron operators. There is no such rule when separately optimized orbitals are used. Now, in principle, all bra-ket determinant pairs contribute to the matrix element between the two multiconfigurational wave functions. A spin singlet fragment wave function obtained with a (small) CAS $(6,6)$ SCF calculation contains 400 determinants (no spatial symmetry is considered). Combining two such fragment wave functions in a NOCI-Fragments study leads to an MEBF with 160000 determinants and, hence, there are in principle $2.56 \times 10^{10}$ determinant pairs that need to be considered to calculate an element of the NOCI matrix. Although many determinant pairs can be safely discarded based on the small product of the CI coefficients, there is still a huge amount of contributions that need to be calculated. Moreover, the evaluation of these contributions to the matrix elements is less straightforward than that in the orthogonal case. Following the outline of Löwdin ${ }^{34}$ on how to calculate the interaction between nonorthogonal determinants, several algorithms have been developed ${ }^{35-46}$ to speed up the procedure as much as possible. We follow here the general nonorthogonal matrix elements (GNOME) algorithm outlined in refs 35, 47, 48. To ensure that the diabatic character of the fragment wave functions is exactly maintained in the MEBFs of the NOCI calculation, we perform a singular value decomposition of the orbitals for each determinant pair of bra- and ket-MEBFs.

Parker and co-workers ${ }^{49}$ introduced an active-space decomposition CASSCF method, ASD-CASSCF, for molecular dimers that allows for the efficient computation of the dimer complete-active-space wave function while only 
constructing the monomer active-space wave functions. Dimer states are formed from linear combinations of direct products of localized orthogonal monomer states, analogous to our MEBFs, and Hamiltonian matrix elements are computed directly without explicitly constructing the product space. This procedure gives rise to problems in covalently linked chromophores that hamper applications of ASD-CASSCF to such systems, but Kim et al. ${ }^{50}$ eliminated this problem by applying orbital optimization. In both approaches, all configurations, including, for example, CT configurations, are expressed in one common set of orthogonal orbitals. If the different MEBFs are expressed in different, mutually nonorthogonal, orbital sets, this procedure is not possible.

Now, one could misdoubt the necessity of using different orbitals to describe the electronic states involved in the phenomenon under study. As illustrated above, state-specific orbital relaxation plays an important role in many cases and describing different electronic states with one set of average orbitals is not always the most appropriate procedure. In the first place, it leads to reference wave functions for dynamic electron correlation treatments that can be far from optimal (see for, example, refs 51-53), making the estimate of the dynamic correlation effect unreliable. Second, expressing the final wave function in a limited number of MEBFs greatly facilitates an intuitive interpretation of the physics of the system.

As reported recently, the calculation of the different contributions to the elements of the NOCI matrix can be efficiently parallelized, leading to a linear scaling of the method with the number of cores. ${ }^{47,48}$ This has set the fundamentals for NOCI calculations on larger systems, but there is still an important bottleneck that needs to be resolved before largescale applications can be run with the above-outlined approach. So far, our NOCI calculations have used an atomic orbital (AO) basis to express the integrals. For efficient handling of the different determinant pairs, these integrals have to be kept in memory, putting rather severe requirements on the computer resources when it comes to anything but the smallest test systems. In ordinary CI calculations with one set of orbitals, the integrals undergo a transformation and are written on the basis of the MOs occupied in any of the configurations considered in the wave function. This drastically reduces the size of the two-electron integrals file, but a similar procedure is not straightforward in the NOCI method because the electronic states involved are described with different sets of MOs. Here, we reduce the integrals file by a transformation from the AO basis set to a new MO-based one-electron basis, common to all states, which is only slightly bigger than the number of MOs with nonzero occupation and therefore enormously reducing the computational cost in comparison with what is needed when the integrals are expressed in the original AO basis (which is, of course, also a common basis for all of the states).

After describing the procedure to construct the common MO basis, we discuss in detail the trade-off between accuracy and computational resources (memory and wall-clock time) of transforming the integrals to this new common basis. To complete the picture, we also report the dependency on the thresholds that are used to decide whether a determinant pair is relevant for the matrix element between two nonorthogonal states. The introduction of a common MO basis and the selection of the determinant pairs based on the product of their
CI coefficients have significantly put forward the limit of application.

\section{GENERATION OF THE REDUCED COMMON MOLECULAR ORBITAL BASIS}

Consider $K$ different electronic states of one of the molecules (or fragments) of the cluster under study. Typically, each molecular state $\Psi_{\mu}$ is described by a multiconfigurational selfconsistent field (MCSCF) wave function expanded in terms of Slater determinants. The determinants themselves can be written in terms of antisymmetrized products of MOs. The MOs are linear combinations of all $\mathrm{N} \mathrm{AO}$ basis functions in the fragment

$$
\left|\phi_{i}^{\mu}\right\rangle=\sum_{j=1}^{N} c_{i j}^{\mu}\left|\chi_{j}\right\rangle
$$

where $\mu$ is one of the electronic states from the set $K$ and $c_{i j}^{\mu}$ are the orbital coefficients optimized for state $\mu$. The MOs of each state are orthogonal among each other, but not necessarily orthogonal to the MOs of other electronic states.

$$
S_{i j}^{\mu \nu}=\left\langle\phi_{i}^{\mu} \mid \phi_{j}^{\nu}\right\rangle= \begin{cases}\delta_{i j} & \text { if } \mu=\nu \\ \sum_{k, l}^{N} c_{k i}^{\mu} c_{l j}^{\nu} s_{k l} & \text { if } \mu \neq \nu\end{cases}
$$

where $s_{k l}$ is the atomic orbital overlap and $c_{k i}^{\mu}$ are the MO coefficients ( $k, l$ run over the AOs and $i, j$ are the MO indices). By grouping together the MOs with nonzero occupation of all $K$ states, a common basis for the given set of electronic states of the molecule can be formed.

$$
\begin{aligned}
& \left\{\phi_{1}^{1}, \phi_{2}^{1}, \ldots, \phi_{n_{1}}^{1}, \phi_{1}^{2}, \phi_{2}^{2}, \ldots, \phi_{n_{2}}^{2}, \phi_{1}^{3}, \phi_{2}^{3}, \ldots, \phi_{n_{3}}^{3},\right. \\
& \left.\quad \ldots, \phi_{1}^{K}, \phi_{2}^{K}, \ldots, \phi_{n_{K}}^{K}\right\}
\end{aligned}
$$

where $\phi_{i}^{\mu}$ is the $i$ th $\mathrm{MO}$ in the $\mu$ th electronic state and $n_{\mu}$ is the number of MOs with nonzero occupation for each state $\mu$. The matrix of $\mathrm{MO}$ coefficients $\mathbf{C}$ has dimensions $(M \times N)$, with $M$ $=\sum_{\mu=1}^{K} n_{\mu}$. Although the orbitals of different electronic states are different, they are still similar enough to make that such a common basis has linear dependencies that need to be removed before further processing. To do so, we first diagonalize the overlap matrix $\mathbf{S}$, dimension $(M \times M)$, of the common MO basis defined in eq 5 .

$$
\mathbf{S}^{\prime}=\mathbf{U}^{\dagger} \mathbf{S U}
$$

The matrix of eigenvectors (U) defines a new set of MOs to describe the electronic states, and linear dependencies are removed by only considering the $L$ vectors whose eigenvalues $\left(\mathbf{S}^{\prime}\right)$ are larger than a given threshold $\left(\tau_{\mathrm{MO}}\right)$.

$$
\mathbf{U} \stackrel{\tau_{\mathrm{MO}}}{\longrightarrow} \mathbf{V}
$$

We now have a reduced common basis $\mathbf{V}$, of dimension $(L \times$ $M)$, from $\mathbf{U}$ of dimension $(M \times M)$, where $L<M$. Next, we express $\mathbf{V}$ in the atomic orbital basis by

$$
\mathbf{B}=\mathbf{C}\left(\mathbf{S}^{\prime \prime}\right)^{-1 / 2} \mathbf{V}
$$

where $\mathbf{S}^{\prime \prime}$ contains the $L$ largest eigenvalues of $\mathbf{S}^{\prime}$ and $\mathbf{B}$ constitutes a set of orthogonal vectors of dimension $L \times N$ for the fragment under consideration. Finally, matrices $\mathbf{D}^{\mu}$ with dimension $\left(n_{\mu} \times L\right)$ are constructed to express the MOs of each electronic state $\mu$ in the common compact basis $\mathbf{B}$. 


$$
\mathbf{D}^{\mu}=\mathbf{B}^{\dagger} \mathbf{s}^{\mu}
$$

where $\mathbf{C}^{\mu}$ is the matrix of MO coefficients for each electronic state $\mu$. In a strictly orthogonal approach with one set of MOs to express all of the electronic states, the $\mathbf{D}^{\mu}$ matrices are unit matrices, but this is no longer the case here. The MOs are written in terms of the new compact basis for the molecule under consideration as

$$
\left|\phi_{i}^{\mu}\right\rangle=\sum_{j=1}^{L} d_{i j}^{\mu}\left|b_{j}\right\rangle
$$

In the standard NOCI approach where only one molecule is considered, this is the end of the transformation procedure. In the case of NOCI-Fragments, the procedure is repeated for all other molecules (fragments) in the system and, after collecting the resulting basis sets of all molecules, the one- and twoelectron integrals are transformed to the common MO basis of the whole cluster. Increasing the value of the threshold $\tau_{\mathrm{MO}}$ leads to a loss of information about the nonorthogonality between the states in the common MO basis. To what extent this affects the elements of the NOCI matrix is the subject of the first part of the Results and Discussion section.

The number of determinant pairs that needs to be considered in the calculation of an element of the NOCI matrix scales roughly with the fourth power of the number of determinants in the CAS used to generate the molecular/ fragment wave functions. This makes the application of any but the smallest CAS prohibitive. There is, however, a simple solution to this bottleneck, realizing that the contribution of each determinant pair in the NOCI matrix element is weighted by the product of the CI coefficients of the two determinants. Hence, the contribution of two determinants, each having a coefficient of the order of $10^{-6}$, is multiplied by $10^{-12}$, which will make it most probably negligible. To explore the possibilities of neglecting a large portion of the determinant pairs, we have introduced a second threshold in the NOCI procedure, $\tau_{\text {det }}$ which eliminates all determinant pairs whose product of CI coefficients is smaller than the threshold.

Before putting the influence of the new thresholds to the test, we first stress that the NOCI implementation based on the GNOME algorithm ${ }^{35,54}$ is not meant to be a general electronic structure method. In fact, from the very beginning, the method is known to perform rather poorly in the description of covalent bonds. ${ }^{55}$ The Supporting Information (SI) summarizes the NOCI-Fragments results on the dissociation of $\mathrm{F}_{2}$ reported in ref 55 . When building the MEBFs of the NOCI from atomic wave functions for $\mathrm{F}^{-} \mathrm{F}^{-}$, and $\mathrm{F}^{+}$, i.e., using the individual atoms as fragments, the energy rises too steeply at short internuclear distances, the equilibrium distance of $\sim 1.7 \AA$ is too long, and the dissociation energy is too small. These inaccuracies are a direct consequence of the way in which the orbitals of the "fragments" are optimized (in the isolated atoms), leading to a large Pauli repulsion between the fragments. A slightly better description is obtained with a standard NOCI calculation where the $\left|. . . \sigma_{\mathrm{g}}^{2}\right|$ and $\left|\ldots \sigma_{\mathrm{u}}^{2}\right|$ determinants (both expressed in their own optimal set of MOs) are used, but with this choice of MEBFs expressed in delocalized MOs, it is better to follow a standard MCSCF approach with these two configurations.

Instead, the NOCI-Fragments method compares very well to other approaches for the calculation of electronic couplings between weakly coupled states. ${ }^{56}$ Furthermore, the direct comparison of the excitation energies in a benzene dimer as a function of the intermolecular distance compares well with a standard CASSCF approach for the dimer, although deviations are getting larger when the two molecules approach each other. Test calculations in which the fragment MOs are optimized in the presence of a frozen density of the other molecule show a reduction of these deviations at a short distance (see Figure S13 of the Supporting Information).

\section{COMPUTATIONAL DETAILS}

The first test case that we considered was the slip-stacked pyridine dimer as depicted in Figure 1. This dimer is small

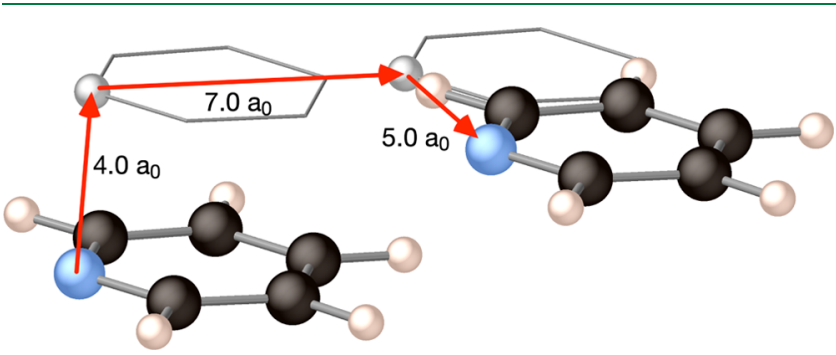

Figure 1. Ball and stick representation of the slip-stack-arranged pyridine dimer. Dark spheres show carbon, blue represents nitrogen, and the lighter spheres show hydrogen.

enough to perform a large set of test calculations within a reasonable time, yet showing the most relevant many-electron states that play a role in intermolecular electron and energy transfer processes. The geometry of the molecule was optimized with B3LYP/6-311G** and the dimer was formed by placing the second unit (with identical internal coordinates) at a perpendicular distance of $4 a_{0}$ to the first and sliding it by $7 a_{0}$ along the $x$-axis and then by $5 a_{0}$ along the $y$-axis. In addition to the ground state $\left(S_{0}\right)$, we also considered the following molecular functions: first excited singlet $\left(S_{1}\right)$, the lowest triplet excited state $\left(\mathrm{T}_{1}\right)$, and the cationic $\left(D_{1}^{+}\right)$and anionic $\left(D_{1}^{-}\right)$states. The optimal MOs of these states $\left(\left|\phi_{\mathrm{i}}^{\mu}\right\rangle\right.$ of eq 3 ) are generated from CASSCF calculations with an active space formed by either four electrons in four active orbitals $(\mathrm{CAS}(4,4))$ or six electrons in six orbitals $(\mathrm{CAS}(6,6))$. The common MO basis (eq 8) was generated with a program written in $\mathrm{f95}$, which also expresses the molecular wave functions in the new basis (eq 9). All of the single-molecule calculations and the transformation of integrals to the common MO basis for the dimer were performed with the GAMESSUK package. ${ }^{57}$

The construction of the MEBFs and the subsequent calculation of the matrix elements were done with the GronOR code $\mathrm{e}^{47,48}$ on the Summit supercomputer at Oak Ridge National Laboratory (Tennessee). The 4608 nodes of this machine are composed of two CPUs (totaling 44 cores) that share 512 GB of memory and six GPUs with 16 GB each. All runs, unless otherwise specified, were run on 32 nodes, each with six ranks per node. GronOR scales linearly with the number of cores up to the machine limit.

Test calculations were done on the following systems following the same procedure as that outlined for the pyridine case: 1-pyridine dimer cc-pVTZ, 2-pyridine dimer 6$311 \mathrm{G}^{* *}$, 3-naphthalene dimer cc-pVDZ, 4-pyridine tetramer 6-311G**, 5-pyridine tetramer cc-pVTZ, and 6tetracene dimer cc-pVDZ. The pyridine tetramer test cases were constructed by placing one pyridine molecule $7 a_{0}$ 
vertically above and one $7 a_{0}$ below the dimer geometry. The results for systems $2-6$ can be found in the Supporting Information. The coordinates of all test systems are given in the Supporting Information.

The test cases have been chosen with the latest applications of our group in mind. ${ }^{56,58-61}$ These focus mainly on inter- and intramolecular electron and energy transfer processes that occur in singlet fission, ${ }^{62-65}$ donor-acceptor charge transfer, ${ }^{66-68}$ and exciton delocalization and dispersion. ${ }^{69-71}$ In all of these cases, several diabatic states with different electronic configurations play a fundamental role and, hence, orbital relaxation is an important factor for obtaining an accurate theoretical description. This organic playground is, of course, not the only area of chemistry where NOCI can be applied; systems with transition metals have also been studied but have usually been restricted to rather small (model) complexes. ${ }^{15,35,72-74}$ The approach presented here will certainly also make the study of more realistic transition metal systems within reach. Roughly speaking, the transformation of integrals to the common MO basis means that our NOCI implementation no longer scales with the size of the atomic orbital basis but rather with the number of electrons in the system, as the dimension of the common MO basis is only slightly larger than half the number of electrons in the system. Different from orthogonal CI approaches, we only need to consider the MOs with nonzero occupation in the integral transformation.

\section{RESULTS AND DISCUSSION}

Figure 2 shows the eigenvalues of the molecular orbital overlap matrix of the pyridine molecule for the five states $S_{0}, S_{1}, T_{1}, D_{1}^{+}$,

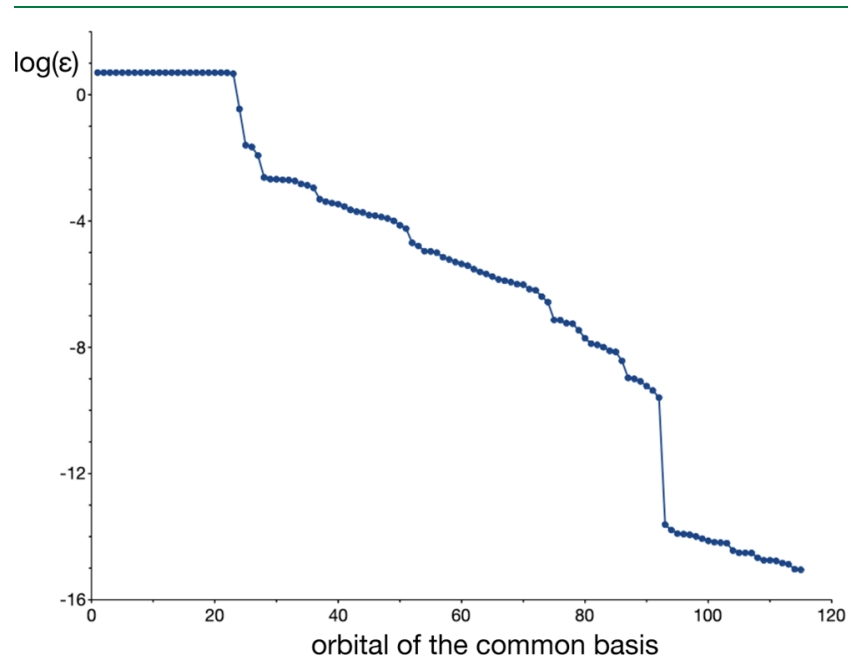

Figure 2. Eigenvalues of the MO overlap matrix of the five electronic states considered for the pyridine molecule.

and $D_{1}^{-}$. The first 23 basis functions of the common MO basis with eigenvalues close to five describe the common part of the occupied (19 inactive and four active) orbitals of the five electronic states. The next functions with smaller eigenvalues (the first 15 are depicted in Figure S12 of the Supporting Information) are responsible for the description of the nonorthogonality, or the difference, in the orbitals of the different electronic states. Although not easily interpreted, basis functions $1-4$ and 6 seem to describe differences in the $\pi$-orbitals of the different electronic states and functions 5, 710 can be considered to take into account possible changes in the $\mathrm{C}-\mathrm{H}$ bonds. It becomes more difficult to assign a role for functions $11-15$.

In fact, the functions with the smallest eigenvalues are just linear dependent counterparts of the basis functions with larger eigenvalues and, hence, going to the right in Figure 2, the functions with eigenvalues smaller than 1 gradually change their role from describing the nonorthogonality between the states to being simple linear dependent vectors not carrying any significant information. In the next section, we determine the optimal value for truncating the common MO basis to remove the linear dependencies but still maintaining the basis flexible enough to express the differences between the electronic states.

4.1. Determination of the Optimal $\tau_{\mathrm{MO}}$. As expected, increasing the value of the threshold for eliminating vectors from the common MO basis increases the difference in the total energies of the MEBFs of the pyridine dimer with respect to the reference values obtained with the AO basis. A roughly linear increase is observed on a double-log plot for all of the systems that we looked at. Thresholds of $10^{-8}$ lead to energy differences of the order of $10^{-8} E_{\mathrm{h}}$, while the much bigger threshold of $10^{-3}$ eliminates so many functions from the basis that the calculated total energy deviates by approximately $10^{-3}$ $E_{\mathrm{h}}$. Such large deviations are, of course, unacceptable if accurate total energies are required, but the total energy is not exactly the property that defines the physics of the system under study. For a better insight into what can be considered as a safe threshold for eliminating functions from the common MO basis, we have to look into other quantities. The first one that we have analyzed is the electronic coupling $V_{i j}$ between the $\mathrm{S}_{0} \mathrm{~S}_{1}$ and $\mathrm{T}_{1} \mathrm{~T}_{1} \mathrm{MEBF}$. These two electronic configurations play a fundamental role in the rationalization of singlet fission $^{58,62,63}$ and the matrix element $\left\langle\mathrm{S}_{0} \mathrm{~S}_{1}|\hat{H}| \mathrm{T}_{1} \mathrm{~T}_{1}\right\rangle$ is a key element in the calculation of the electronic coupling $V_{i j}$ between the two configurations:

$$
V_{i j}=\frac{\left\langle\Psi_{i}|\hat{H}| \Psi_{j}\right\rangle-\frac{\left(\left\langle\Psi_{i}|\hat{H}| \Psi_{i}\right\rangle+\left\langle\Psi_{j}|\hat{H}| \Psi_{j}\right\rangle\right)}{2} \cdot\left\langle\Psi_{i} \mid \Psi_{j}\right\rangle}{1-\left\langle\Psi_{i} \mid \Psi_{j}\right\rangle^{2}}
$$

Figure 3 compares $V_{i j}$ obtained with the $\mathrm{AO}$ basis to those calculated with the common MO basis, varying the threshold

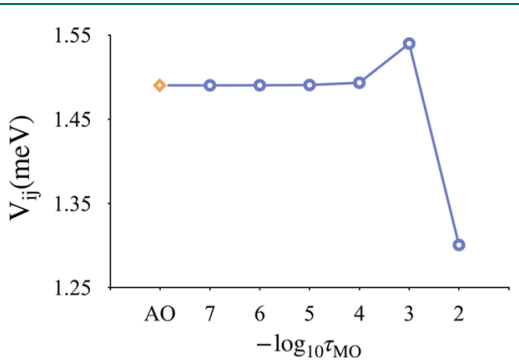

Figure 3. Electronic coupling $V_{i j}\left(\right.$ in $\mathrm{meV}$ ) between $\left|\mathrm{S}_{0} \mathrm{~S}_{1}\right\rangle$ and $\left|\mathrm{T}_{1} \mathrm{~T}_{1}\right\rangle$ for the pyridine dimer test system as a function of the threshold $\tau_{\mathrm{MO}}$ for removing basis functions from the common $\mathrm{MO}$ basis. $\mathrm{AO}$ refers to the value obtained with the $\mathrm{AO}$ basis.

for linear dependencies. Pyridine $\operatorname{CAS}(4,4)$ molecular wave functions with the cc-pVTZ basis set were used to construct the MEBFs. Moving to the right along the $\tau_{\mathrm{MO}}$ axis, more basis functions are eliminated from the common MO basis with an increasing threshold. 
Although the coupling is much smaller than that in systems with singlet fission properties, for example, the calculated NOCI-Fragments coupling for a tetracene dimer is around 50 $\mathrm{meV},{ }^{56}$ it allows us to draw conclusions about the dependence of the coupling on the threshold. It is seen that for $\tau_{\mathrm{MO}}$ smaller than or equal to $10^{-4}$, the coupling is exactly the same as the one calculated with the $\mathrm{AO}$ basis. A small deviation of less than $0.1 \mathrm{meV}$ is found when $\tau_{\mathrm{MO}}=10^{-3}$ and, even with the $10^{-2}$ threshold, a reasonable coupling is obtained. From Figure 4, it

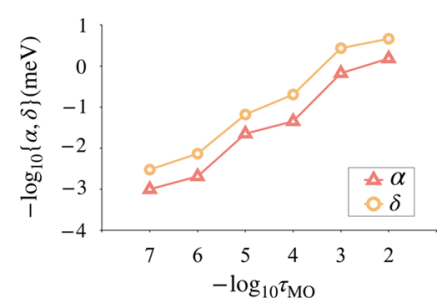

Figure 4. Mean absolute error $(\alpha)$ and maximum absolute deviation $(\delta)$ (both in $\mathrm{meV}$ ) of the electronic couplings among the five MEBFs of the NOCI with respect to the values obtained with the AO basis.

can be noted that this behavior is general to all 15 off-diagonal elements of the NOCI matrix. The mean absolute error $(\alpha)$ and the maximum absolute deviation $(\delta)$ in the electronic coupling plotted in the figure are calculated with the corresponding values in the $\mathrm{AO}$ basis as a reference. Negligible differences are observed up to $\tau_{\mathrm{MO}}=10^{-3}$, and increasing the threshold by a factor of 10 introduces small deviations.

Having established that the results of the AO basis can be reproduced with a common $\mathrm{MO}$ basis, we now focus on the computational savings. Keeping the number of nodes and other computational parameters constant, we have compared the wall-clock time and the size of the integral file for the calculation of the 21 unique elements in the $6 \times 6 \mathrm{NOCI}$ matrix for different values of $\tau_{\mathrm{MO}}$.

Figure 5 shows a dramatic drop in the computer time when the $\mathrm{AO}$ basis is replaced by the common $\mathrm{MO}$ basis with $\tau_{\mathrm{MO}}=$

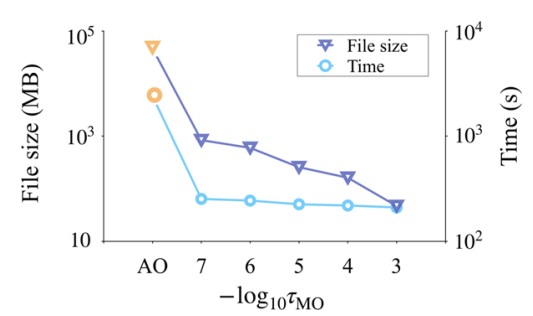

Figure 5. Integral file size in $\mathrm{MB}$ (left) and computer time in seconds (right) as functions of the threshold $\tau_{\mathrm{MO}}$.

$10^{-7}$. The use of larger thresholds only slightly reduces the time. On smaller machines, the decrease in the computer time continues to larger thresholds as can be seen in Figure 6.

The size of the integral file and, hence, the memory requirements decrease more steadily as functions of the threshold and are about 3 orders of magnitude smaller for $\tau_{\mathrm{MO}}$ $=10^{-3}$ than that for the integral file using the $\mathrm{AO}$ basis. In comparison to the original $\mathrm{AO}$ basis of 570 basis functions, the maximum size of the common $\mathrm{MO}$ basis set is 230: 10 electronic states $\left(S_{0}, S_{1}, T_{1}, D_{1}^{+}\right.$, and $D_{1}^{-}$on both molecules), each with 19 inactive +4 active $=23$ occupied orbitals. This is already reduced to 148 basis functions by applying a threshold

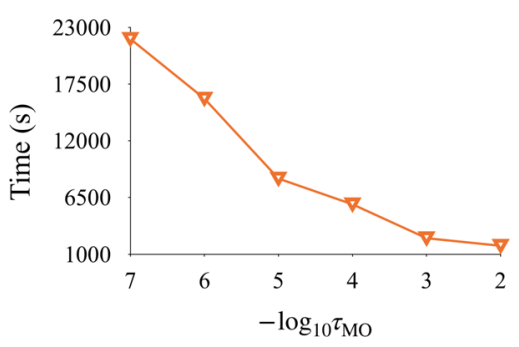

Figure 6. Computer wall-clock time (in seconds) as a function of $\tau_{\mathrm{MO}}$ when performed using two GPUs on the computer cluster.

of $10^{-7}$, and less than one-third of the basis functions are retained when $\tau_{\mathrm{MO}}$ increases to $10^{-3}$.

The last element that we checked was the performance of the common $\mathrm{MO}$ basis to reproduce the relative energies of the MEBFs taking the $\mathrm{S}_{0} \mathrm{~S}_{0}$ configuration as the reference energy. The first feature that strikes the eye in Figure 7 is the

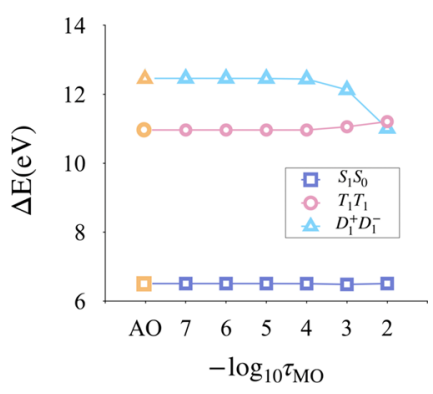

Figure 7. Excitation energies $\Delta E$ (in $\mathrm{eV}$ ) for three states calculated using different $\tau_{\mathrm{MO}}$ values. AO refers to the value obtained with the AO basis.

fact that the pyridine dimer is definitely not a candidate to show singlet fission. The $\mathrm{T}_{1} \mathrm{~T}_{1}$ configuration lies way too high in energy with respect to the $S_{0} S_{1}$ state, while they should be close in energy for singlet fission. More important for the present study is that the relative energies are not affected by the change from the AO basis to the common MO basis unless the threshold for linear dependencies is very high, although the differences with the $\mathrm{AO}$ basis are marginal even for $\tau_{\mathrm{MO}}=10^{-3}$.

We performed the same set of tests for the other molecules mentioned in the computational details and obtained the same trend in the results. The mean errors $\alpha$ and maximum deviations $\delta$ in electronic coupling given in Figure 8 are small in all cases, except for the tetracene dimer (6), for which we observe a deviation of around $10 \mathrm{meV}$ in a coupling that is as

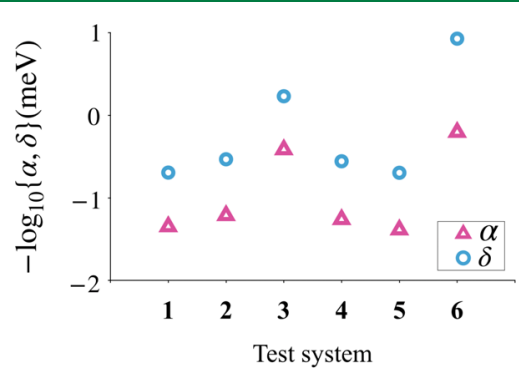

Figure 8. Mean absolute error $(\alpha)$ and maximum absolute deviation $(\delta)$ (both in $\mathrm{meV}$ ) of the electronic couplings among the five MEBFs of the NOCI with respect to the values obtained with the AO basis, with $\tau_{\mathrm{MO}}=10^{-4}$ for the six different test systems. 
large as $77 \mathrm{meV}$ in the reference calculation (see SI). From the ratios of integral file sizes depicted in Figure 9, we can see that

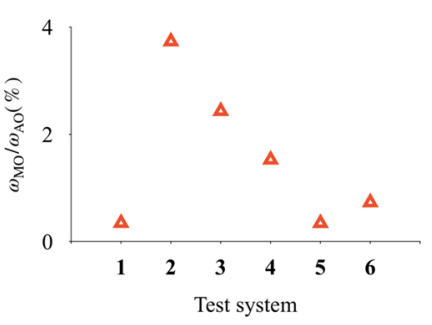

Figure 9. Ratio of the integral file size (in \%) in the MO basis $\left(\omega_{\mathrm{MO}}\right)$ and the AO basis $\left(\omega_{\mathrm{AO}}\right)$ for the test systems at $\tau_{\mathrm{MO}}=10^{-4}$.

the integral file size is reduced by approximately 2 orders of magnitude in all systems, which is especially important for calculations on larger systems. Finally, the speed-up (reduction of the wall-clock time) is also close to 2 orders of magnitude in systems 1, 3, and 4. For system 2, we find a slightly smaller reduction because this system is rather small anyway and the impact of expressing the integrals in the common MO basis is not so well seen in the large-scale computer used in this study. For systems 5 and $\mathbf{6}$, we have not been able to perform the NOCI calculation in the AO basis due to the excessive size of the integral file (reference calculations for $\alpha$ and $\delta$ are the ones with $\left.\tau_{\mathrm{MO}}=10^{-9}\right)$. The reduced number of basis functions implies shorter summations in the evaluation of nonorthogonal matrix elements compared to the $\mathrm{AO}$ basis. The reduced common $\mathrm{MO}$ basis also now makes it possible to have multiple ranks processing the integrals in GPUs for large systems, hence accelerating the calculation even more.

To close the discussion of the influence of the truncation of the common MO basis, we would like to stress that the analysis of the underlying physics of the system under study is not done at the one-electron level in our implementation of NOCI but rather at the many-electron level by looking at the relative importance of the different MEBFs in the final N-electron wave functions obtained by diagonalizing the NOCI matrix. Since the initial diabatic character of the functions is strictly maintained during the calculation, the coefficients in the NOCI wave function give a direct indication of the importance of the MEBFs, which can univocally be assigned to physical concepts such as charge transfer, local excitation, relaxed hole states, etc. The threshold for reducing the common MO basis must be chosen such that only the linear dependencies are removed from the basis. Making the threshold too large will lead to a removal of functions that are needed to account for the nonorthogonality between the different electronic states and, therefore, too large a value of $\tau_{\mathrm{MO}}$ will lead to a basis that is not flexible enough for a proper description of the fragment wave functions.

4.2. Selection of Determinant Pairs. Starting again with the matrix element between the $T_{1} T_{1}$ and $S_{0} S_{1}$ states of the pyridine dimer $\left(\mathrm{CAS}(6,6) / 6-311 \mathrm{G}^{* *}\right)$ with the integrals already expressed using a common $\mathrm{MO}$ basis with $\tau_{\mathrm{MO}}=$ $10^{-4}$, Figure 10 shows how the size of the coupling varies with increasing determinant pair threshold $\tau_{\text {det }}$.

The calculated values are virtually the same for $\tau_{\text {det }}$ smaller than or equal to $10^{-5}$. For larger thresholds, we see very small deviations and filtering out all determinant pairs with a CI coefficient product smaller than $10^{-3}$ affects the NOCI matrix element by not more than $0.3 \mathrm{meV}$. Figure 11 compares the

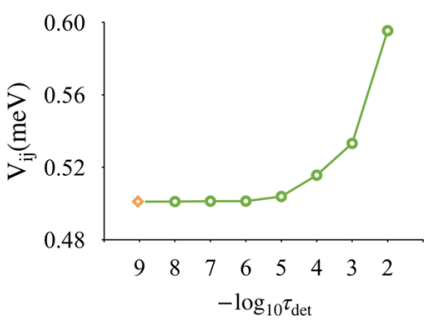

Figure 10. Electronic coupling $V_{i j}$ (in $\mathrm{meV}$ ) between $\left|\mathrm{S}_{0} \mathrm{~S}_{1}\right\rangle$ and $\mid$ $\left.\mathrm{T}_{1} \mathrm{~T}_{1}\right\rangle$ as a function of the determinant threshold $\tau_{\text {det. }}$.

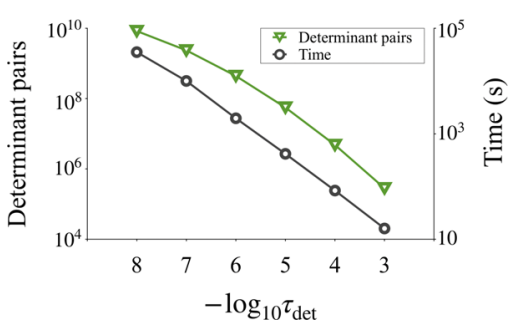

Figure 11. Number of determinant pairs that were used to construct the NOCI matrix and the time for the NOCI calculation (in seconds) as functions of $\tau_{\mathrm{det}}$.

number of determinant pairs that were considered in the calculation of these matrix elements to the time it took to process all of the pairs and calculate the matrix elements for the NOCI matrix.

These calculations were performed on 256 Summit nodes with six ranks per node and show how the number of determinant pairs decreases steadily with increasing $\tau_{\text {det }}$. Taking a threshold of $10^{-4}$, the reduction in the number of determinant pairs is almost 4 orders of magnitude compared to the smallest threshold that we have used. From Figure 10, it can be seen that using a threshold of $10^{-4}$ does not significantly affect the calculated coupling, but reduces the computer time by more than 2 orders of magnitude Figure 11 .

The same behavior is observed for the other matrix elements. Figure 12 represents the mean absolute error and

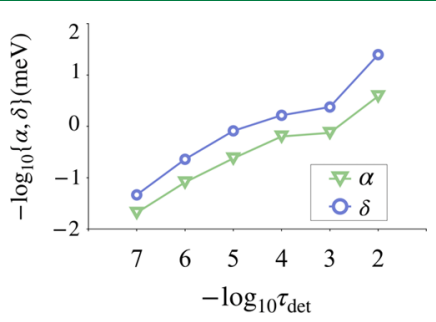

Figure 12. Mean absolute error $(\alpha)$ and maximum absolute deviation $(\delta)$ (both in $\mathrm{meV}$ ) of the electronic couplings among the five MEBFs of the NOCI with respect to $\tau_{\text {det }}=10^{-9}$, which was the smallest value that we chose to examine.

the maximum absolute deviation (both in $\mathrm{meV}$ ) as functions of $\tau_{\text {det }}$. The results remain basically stable up to $\tau_{\text {det }}=10^{-5}$; for higher thresholds, we start to observe small deviations. The excited state energies in Figure 13 are hardly sensitive to the value of the threshold; even with a value of $10^{-3}$, the changes are minimal.

The figures show that up to $\tau_{\text {det }}=10^{-5}$, there is nearly no loss in accuracy, while the computational time is drastically reduced. The introduction of the threshold for determinant 


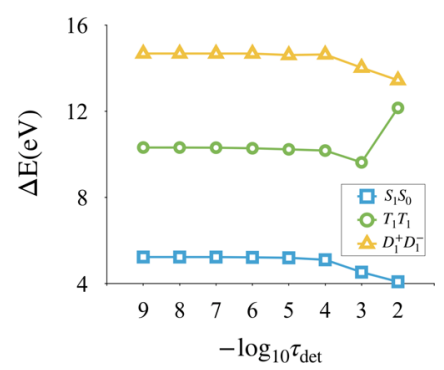

Figure 13. Excitation energies $\Delta E$ (in $\mathrm{eV}$ ) for three states calculated using different $\tau_{\text {det }}$ values.

pairs $\tau_{\text {det }}$ makes the NOCI much less dependent on the size of the active space employed for the calculation of the molecular wave functions. As illustrated in Figure S11 of the Supporting Information, the largest part of determinants introduced by enlarging the active space turn out to have rather small coefficients in the CAS wave function. These are effectively filtered out by the threshold on the determinant pairs and although the calculation time of the NOCI matrix becomes longer when a larger CAS is applied in the fragment, the increase is much less prominent than the quartic dependence $\left(\mathrm{N}^{4}\right.$, with $\mathrm{N}$ being the number of determinants in the fragment CAS wave function) that can be expected without filtering.

This one case study is likely not enough to establish $\tau_{\text {det }}=$ $10^{-5}$ as a universally applicable threshold for all systems, but this very straightforward method used to reduce the number of determinant pairs that needs to be evaluated in the calculation of the matrix elements clearly pushes forward the border of applicability of the NOCI method. For a more general approach, it is most probably unavoidable to leave behind the complete active space expansion of the fragment functions. We are currently investigating the use of the ICE-CI approach developed by Neese and co-workers based on the CIPSI approach of Huron, Malrieu, and Rancurel. ${ }^{75}$ In this approach, the multiconfigurational wave function is constructed iteratively to only include the most important configurations. Hence, the final wave function does not, by definition, contain any "configurational deadwood".

\section{CONCLUSIONS}

In a NOCI, each state is expressed in its own optimal molecular orbital set and this complicates the transformation of the integrals to an MO basis, commonly applied in configuration interaction calculations with one set of orthogonal MOs. Instead, we have introduced a common MO basis constructed by grouping together all of the MOs of the different electronic states, followed by an orthogonalization and the removal of the linear dependencies through a threshold that can be varied to find the optimal trade-off between accuracy and computational cost.

The introduction of a common MO basis set to express the wave functions of the different electronic states effectively reduces the computational cost of NOCI calculations. The transformation of the one- and two-electron integrals to this common MO basis drastically reduces the size of the integral files and allows to keep the integrals in memory, either using the shared memory of a group of CPUs (GPUs), or multiple copies, each on a single CPU (GPU), depending on the amount of memory available. Furthermore, the reduced number of basis functions in the common $\mathrm{MO}$ basis makes the summations in the calculation of the nonorthogonal matrix elements much shorter than that for the $\mathrm{AO}$ basis that has been applied so far in NOCI.

Test calculations on the pyridine dimer (and on the larger systems presented in the Supporting Information) show that the threshold for removing the linear dependencies in the common MO basis $\left(\tau_{\mathrm{MO}}\right)$ can be safely taken to be $10^{-4}$ and, if needed, even 10 times larger to generate some additional savings (for example, on smaller computers). A consequence of rewriting the integrals in the common MO basis is that it has become much more efficient to apply AO basis sets of good quality in the calculations of the fragments, as the NOCI cost no longer directly scales with the size of the AO basis but rather with the number of electrons.

The second important bottleneck in the NOCI is the huge number of matrix elements over determinant pairs that needs to be evaluated for the calculation of a single matrix element over MEBFs. The introduction of a second parameter in the method, $\tau_{\mathrm{det}}$ allows the consideration of much larger active spaces in the fragment calculations. The $\tau_{\text {det }}$ parameter removes all of the determinant pairs whose product of CI coefficients in the MEBFs is smaller than the threshold and effectively removes what is sometimes called the configurational "deadwood". While the values of the matrix elements of MEBFs do not critically depend on the threshold for the linear dependency in the common basis (unless very large thresholds are used), the screening of the determinant pairs has a larger impact on the results. A threshold of $10^{-5}$ turns out to be the largest one for reliable matrix elements, but checking with smaller (or larger) thresholds is advised.

\section{ASSOCIATED CONTENT}

\section{Supporting Information}

The Supporting Information is available free of charge at https://pubs.acs.org/doi/10.1021/acs.jctc.9b01144.

Cartesian coordinates, electronic couplings, and excitation energies of the test systems; number of determinants versus number of active orbitals; graphs of the common molecular orbitals; $\mathrm{F}_{2}$ dissociation; and benzene dimer excitation energies as functions of the intermolecular distance (PDF)

\section{AUTHOR INFORMATION}

\section{Corresponding Authors}

Coen de Graaf - Department of Physical and Inorganic Chemistry, Universitat Rovira i Virgili, 43007 Tarragona, Spain; Theoretical Chemistry Group, Zernike Institute for Advanced Materials, University of Groningen, 9747 AG Groningen, The Netherlands; ICREA, 08010 Barcelona, Spain; ○ orcid.org/0000-0001-8114-6658; Email: coen.degraaf@ urv.cat

Remco W. A. Havenith - Theoretical Chemistry Group, Zernike Institute for Advanced Materials and Stratingh Institute for Chemistry, University of Groningen, 9747 AG Groningen, The Netherlands; Ghent Quantum Chemistry Group, Department of Inorganic and Physical Chemistry, Ghent University, 9000 Gent, Belgium; 이이.org/0000-00030038-6030; Email: r.w.a.havenith@rug.nl

\section{Authors}

R. K. Kathir - Theoretical Chemistry, Zernike Institute for Advanced Materials, University of Groningen, 9747 AG 
Groningen, The Netherlands; (1) orcid.org/0000-0002-48808629

Ria Broer - Theoretical Chemistry Group, Zernike Institute for Advanced Materials, University of Groningen, 9747 AG Groningen, The Netherlands; (1) orcid.org/0000-0002-54379509

Complete contact information is available at:

https://pubs.acs.org/10.1021/acs.jctc.9b01144

\section{Notes}

The authors declare no competing financial interest.

\section{ACKNOWLEDGMENTS}

The authors acknowledge Dr. T. P. Straatsma (Oak Ridge National Laboratory) for technical support, fruitful discussions, and for the calculations on the $\mathrm{F}_{2}$ molecule. This work is part of the research program "Computational sciences for energy research" (project 15CSER73), which is financed by the Dutch Research Council (NWO). This research was funded by the Spanish Ministerio de Economía y Competitividad and the European Regional Development Fund (FEDER), grant number CTQ201783566-P, and by the Generalitat de Catalunya, grant number 2017SGR629. This research used resources (Summit) of the Oak Ridge Leadership Computing Facility at the Oak Ridge National Laboratory, which is supported by the Office of Science of the U.S. Department of Energy under Contract No. DE-AC05-00OR22725 (DD and ESP).

\section{REFERENCES}

(1) Guéron, M.; Shulman, R. G.; Eisinger, J. Energy transfer in dinucleotides. Proc. Natl. Acad. Sci. U.S.A. 1966, 56, 814-818.

(2) Coropceanu, V.; Cornil, J.; da Silva Filho, D. A.; Olivier, Y.; Silbey, R.; Brédas, J.-L. Charge Transport in Organic Semiconductors. Chem. Rev. 2007, 107, 926-952.

(3) Delgado, J. L.; Bouit, P.-A.; Filippone, S.; Herranz, M. A.; Martin, N. Organic photovoltaics: a chemical approach. Chem. Commun. 2010, 46, 4853-4865.

(4) Congreve, D. N.; Lee, J.; Thompson, N. J.; Hontz, E.; Yost, S. R.; Reusswig, P. D.; Bahlke, M. E.; Reineke, S.; Van Voorhis, T.; Baldo, M. A. External Quantum Efficiency Above $100 \%$ in a Singlet-ExcitonFission-Based Organic Photovoltaic Cell. Science 2013, 340, 334337.

(5) Hohenberg, P.; Kohn, W. Inhomogeneous Electron Gas. Phys. Rev. 1964, 136, B864-B871.

(6) Kohn, W.; Sham, L. J. Self-Consistent Equations Including Exchange and Correlation Effects. Phys. Rev. 1965, 140, A1133A1138.

(7) Sinanoğlu, O. Many-Electron Theory of Atoms and Molecules. I. Shells, Electron Pairs vs Many-Electron Correlations. J. Chem. Phys. 1962, 36, 706-717.

(8) Č́žek, J. On the Correlation Problem in Atomic and Molecular Systems. Calculation of Wavefunction Components in Ursell-Type Expansion Using Quantum-Field Theoretical Methods. J. Chem. Phys. 1966, 45, 4256-4266.

(9) Bartlett, R. J.; Musial, M. Coupled-cluster theory in quantum chemistry. Rev. Mod. Phys. 2007, 79, 291.

(10) Hinze, J. MC-SCF. 1. The multi-configuration self-consistentfield method. J. Chem. Phys. 1973, 59, 6424-6432.

(11) Roos, B. O.; Taylor, P. R.; Siegbahn, P. E. A complete active space SCF method (CASSCF) using a density matrix formulated super-CI approach. Chem. Phys. 1980, 48, 157-173.

(12) Olsen, J. The CASSCF method: A perspective and commentary. Int. J. Quantum Chem. 2011, 111, 3267-3272.
(13) Shaik, S. S.; Hiberty, P. C. A Chemist's Guide to Valence Bond Theory; John Wiley \& Sons, 2007.

(14) Except for full CI, where the shape of the orbitals is irrelevant. (15) Olsen, J. Novel methods for configuration interaction and orbital optimization for wave functions containing non-orthogonal orbitals with applications to the chromium dimer and trimer. J. Chem. Phys. 2015, 143, No. 114102.

(16) Kähler, S.; Olsen, J. Non-orthogonal internally contracted multi-configurational perturbation theory (NICPT): Dynamic electron correlation for large, compact active spaces. J. Chem. Phys. 2017, 147, No. 174106.

(17) Kähler, S.; Olsen, J. Dynamic correlation for non-orthogonal reference states: Improved perturbational and variational methods. J. Chem. Phys. 2018, 149, No. 144104.

(18) Høyvik, I.-M.; Olsen, J.; Jørgensen, P. Generalising localisation schemes of orthogonal orbitals to the localisation of non-orthogonal orbitals. Mol. Phys. 2017, 115, 16-25.

(19) Mayhall, N. J.; Horn, P. R.; Sundstrom, E. J.; Head-Gordon, M. Spin-flip non-orthogonal configuration interaction: a variational and almost black-box method for describing strongly correlated molecules. Phys. Chem. Chem. Phys. 2014, 16, 22694-22705.

(20) Sundstrom, E. J.; Head-Gordon, M. Non-orthogonal configuration interaction for the calculation of multielectron excited states. J. Chem. Phys. 2014, 140, No. 114103.

(21) Yost, S. R.; Head-Gordon, M. Size consistent formulations of the perturb-then-diagonalize Møller-Plesset perturbation theory correction to non-orthogonal configuration interaction. J. Chem. Phys. 2016, 145, No. 054105.

(22) Yost, S. R.; Head-Gordon, M. Efficient Implementation of NOCI-MP2 Using the Resolution of the Identity Approximation with Application to Charged Dimers and Long C-C Bonds in Ethane Derivatives. J. Chem. Theory Comput. 2018, 14, 4791-4805.

(23) Oosterbaan, K. J.; White, A. F.; Head-Gordon, M. Nonorthogonal configuration interaction with single substitutions for the calculation of core-excited states. J. Chem. Phys. 2018, 149, No. 044116.

(24) Oosterbaan, K. J.; White, A. F.; Head-Gordon, M. NonOrthogonal Configuration Interaction with Single Substitutions for Core-Excited States: An Extension to Doublet Radicals. J. Chem. Theory Comput. 2019, 15, 2966-2973.

(25) Chen, Z.; Chen, X.; Wu, W. Nonorthogonal orbital based Nbody reduced density matrices and their applications to valence bond theory. I. Hamiltonian matrix elements between internally contracted excited valence bond wave functions. J. Chem. Phys. 2014, 138, No. 164119.

(26) Chen, Z.; Chen, X.; Wu, W. Nonorthogonal orbital based Nbody reduced density matrices and their applications to valence bond theory. II. An efficient algorithm for matrix elements and analytical energy gradients in VBSCF method. J. Chem. Phys. 2014, 138, No. 164120.

(27) Chen, Z.; Chen, X.; Ying, F.; Gu, J.; Zhang, H.; Wu, W. Nonorthogonal orbital based n-body reduced density matrices and their applications to valence bond theory. III. Second-order perturbation theory using valence bond self-consistent field function as reference. J. Chem. Phys. 2014, 141, No. 134118.

(28) Chen, X.; Chen, Z.; Wu, W. Nonorthogonal orbital based Nbody reduced density matrices and their applications to valence bond theory. IV. The automatic implementation of the Hessian based VBSCF method. J. Chem. Phys. 2014, 141, No. 194113.

(29) Burton, H. G. A.; Gross, M.; Thom, A. J. W. Holomorphic Hartree-Fock Theory: The Nature of Two-Electron Problems. J. Chem. Theory Comput. 2018, 14, 607-618.

(30) Burton, H. G. A.; Thom, A. J. W. General Approach for Multireference Ground and Excited States Using Nonorthogonal Configuration Interaction. J. Chem. Theory Comput. 2019, 15, 48514861.

(31) Nite, J.; Jiménez-Hoyos, C. A. Low-Cost Molecular Excited States from a State-Averaged Resonating Hartree-Fock Approach. J. Chem. Theory Comput. 2019, 15, 5343-5351. 
(32) Fukutome, H. Theory of Resonating Quantum Fluctuations in a Fermion System-Resonating Hartree-Fock Approximation. Prog. Theor. Phys. 1988, 80, 417-432.

(33) Hiscock, H. G.; Thom, A. J. W. Holomorphic Hartree-Fock Theory and Configuration Interaction. J. Chem. Theory Comput. 2014, 10, 4796-4800.

(34) Löwdin, P.-O. Quantum theory of many-particle systems. I. Physical interpretations by means of density matrices, natural spinorbitals, and convergence problems in the method of configurational interaction. Phys. Rev. 1955, 97, 1474.

(35) Broer, R.; Nieuwpoort, W. Broken orbital symmetry and the description of valence hole states in the tetrahedral $\left[\mathrm{CrO}_{4}\right]^{2-}$ anion. Theor. Chim. Acta 1988, 73, 405-418.

(36) Prosser, F.; Hagstrom, S. On the rapid computation of matrix elements. Int. J. Quantum Chem. 1968, 2, 89-99.

(37) Gallup, G. Valence-Bond calculations and matrix elements between two tableau functions of non-orthogonal orbitals. Int. J. Quantum Chem. 1972, 6, 899-909.

(38) Raimondi, M.; Simonetta, M.; Tantardini, G. Ab initio valence bond theory. Comput. Phys. Rep. 1985, 2, 171-216.

(39) Verbeek, J.; Van Lenthe, J. H. On the evaluation of nonorthogonal matrix elements. J. Mol. Struct.: THEOCHEM 1991, 229, 115-137.

(40) Leasure, S.; Balint-Kurti, G. Valence-bond theory and the evaluation of electronic energy matrix elements between nonorthogonal Slater determinants. Phys. Rev. A 1985, 31, 2107.

(41) Li, X.; Zhang, Q. Bonded tableau unitary group approach to the many-electron correlation problem. Int. J. Quantum Chem. 1989, 36, 599-632.

(42) McWeeny, R. A spin-free form of valence bond theory. Int. J. Quantum Chem. 1988, 34, 25-36.

(43) Li, J.; Wu, W. New algorithm for nonorthogonal ab initio valence-bond calculations. Theor. Chim. Acta. 1994, 89, 105-121.

(44) Li, J.; McWeeny, R. VB2000: Pushing valence bond theory to new limits. Int. J. Quantum Chem. 2002, 89, 208-216.

(45) Gerratt, J.; Raimondi, M. The spin-coupled valence bond theory of molecular electronic structure. I. Basic theory and application to the ${ }^{2} \sum^{+}$states of BeH. Proc. R. Soc. London, Ser. A 1980, 371, 525-552.

(46) Cooper, D.; Gerratt, J.; Raimondi, M. Modern valence bond theory. Adv. Chem. Phys 1987, 69, 319-397.

(47) Straatsma, T. P.; Broer, R.; Faraji, S.; Havenith, R. W. A. GronOR Nonorthogonal Configuration Interaction Calculations at Exascale. Annu. Rep. Comput. Chem. 2018, 14, 77-91.

(48) Straatsma, T. P.; Broer, R.; Faraji, S.; Havenith, R. W. A.; Aguilar Suarez, L. E.; Kathir, R. K.; Wibowo, M.; de Graaf, C. GronOR: Massively Parallel and GPU-Accelerated Non-Orthogonal Configuration Interaction for Large Molecular Systems. J. Chem. Phys. 2020, 152, No. 064111.

(49) Parker, S. M.; Seideman, T.; Ratner, M. A.; Shiozaki, T. Communication: Active-space decomposition for molecular dimers. $J$. Chem. Phys. 2013, 139, No. 021108.

(50) Kim, I.; Parker, S. M.; Shiozaki, T. Orbital Optimization in the Active Space Decomposition Model. J. Chem. Theory Comput. 2015, 11, 3636-3642.

(51) Domingo, A.; Carvajal, M. A.; de Graaf, C.; Sivalingam, K.; Neese, F.; Angeli, C. Metal-to-metal charge-transfer transitions: reliable excitation energies from ab initio calculations. Theor. Chem. Acc. 2012, 131, 1264.

(52) Meyer, B.; Domingo, A.; Krah, T.; Robert, V. Charge transfer processes: the role of optimized molecular orbitals. Dalton Trans. 2014, 43, 11209-11215.

(53) Domingo, A.; Angeli, C.; de Graaf, C.; Robert, V. Electronic Reorganization Triggered by Electron Transfer: The Intervalence Charge Transfer of a $\mathrm{Fe}^{3+} / \mathrm{Fe}^{2+}$ Bimetallic Complex. J. Comput. Chem. 2015, 36, 861-869.

(54) Broer, R.; Nieuwpoort, W. C. Broken orbital symmetry and the description of hole states in tetrahedral $\left[\mathrm{CrO}_{4}\right]^{2-}$ anion. I.
Introductory considerations and calculations on oxygen 1s hole states. Chem. Phys. 1981, 54, 291-303.

(55) Broer, R. Localized Orbitals and Broken Simmetry in Molecules. Theory and Applications to the Chromate Ion and paraBenzoquinone. Ph.D. Thesis; University of Groningen, 1981.

(56) Aguilar Suarez, L. E.; Kathir, R. K.; Siagri, E.; Havenith, R. W. A.; Faraji, S. Determination of electronic couplings in the singlet fission process using a nonorthogonal configuration interaction approach. Adv. Quantum Chem. 2019, 263.

(57) Guest, M. F.; Bush, I. J.; van Dam, H. J. J.; Sherwood, P.; Thomas, J. M. H.; van Lenthe, J. H.; Havenith, R. W. A.; Kendrick, J. The GAMESS-UK electronic structure package: algorithms, developments and applications. Mol. Phys. 2005, 103, 719-747.

(58) Havenith, R. W. A.; de Gier, H. D.; Broer, R. Explorative computational study of the singlet fission process. Mol. Phys. 2012, $110,2445-2454$.

(59) Wibowo, M.; Broer, R.; Havenith, R. W. A. A rigorous nonorthogonal configuration interaction approach for the calculation of electronic couplings between diabatic states applied to singlet fission. Comput. Theor. Chem. 2017, 1116, 190-194.

(60) Zaykov, A.; Felkel, P.; Buchanan, E. A.; Jovamovic, M.; Havenith, R. W. A.; Kathir, R. K.; Broer, R.; Havlas, Z.; Michl, J. Singlet Fission Rate: Optimized Packing of a Molecular Pair. Ethylene as a Model. J. Am. Chem. Soc. 2019, 141, 17729-17743.

(61) Ryerson, J. L.; Zaykov, A.; Aguilar Suarez, L. E.; Havenith, R. W. A.; Stepp, B. R.; Dron, P. I.; Kaleta, J.; Akdag, A.; Teat, S. J.; Magnera, T. F.; Miller, J. R.; Havlas, Z.; Broer, R.; Faraji, S.; Michl, J.; Johnson, J. C. Structure and photophysics of indigoids for singlet fission: Cibalackrot. J. Chem. Phys. 2019, 151, No. 184903.

(62) Smith, M. B.; Michl, J. Singlet Fission. Chem. Rev. 2010, 110, 6891-6936.

(63) Smith, M. B.; Michl, J. Recent advances in singlet fission. Annu. Rev. Phys. Chem. 2013, 64, 361-386.

(64) Casanova, D. Theoretical modeling of singlet fission. Chem. Rev. 2018, 118, 7164-7207.

(65) Farag, M. H.; Krylov, A. I. Singlet Fission in Perylenediimide Dimers. J. Phys. Chem. C 2018, 122, 25753-25763.

(66) Grabowski, Z. R.; Rotkiewicz, K.; Rettig, W. Structural Changes Accompanying Intramolecular Electron Transfer: Focus on Twisted Intramolecular Charge-Transfer States and Structures. Chem. Rev. 2003, 103, 3899-4031.

(67) Yamazaki, Y.; Takeda, H.; Ishitani, O. Photocatalytic reduction of $\mathrm{CO}_{2}$ using metal complexes. J. Photochem. Photobiol., C 2015, 25, $106-137$.

(68) Oberhofer, H.; Reuter, K.; Blumberger, J. Charge Transport in Molecular Materials: An Assessment of Computational Methods. Chem. Rev. 2017, 117, 10319-10357.

(69) Zimmerman, P. M.; Zhang, Z.; Musgrave, C. B. Singlet fission in pentacene through multi-exciton quantum states. Nature Chem. 2010, 2, 648-652.

(70) Zimmerman, P. M.; Bell, F.; Casanova, D.; Head-Gordon, M. Mechanism for Singlet Fission in Pentacene and Tetracene: From Single Exciton to Two Triplets. J. Am. Chem. Soc. 2011, 133, 1994419952.

(71) Mewes, S. A.; Plasser, F.; Krylov, A. I.; Dreuw, A. Benchmarking Excited-State Calculations Using Exciton Properties. J. Chem. Theory Comput. 2018, 14, 710-725.

(72) de Graaf, C.; Broer, R.; Nieuwpoort, W. C.; Bagus, P. S. On the role of relaxed charge-transfer excitations: $\mathrm{Ni} 3 \mathrm{~s}$ hole states in $\mathrm{NiO}$. Chem. Phys. Lett. 1997, 272, 341-346.

(73) van Oosten, A. B.; Broer, R.; Nieuwpoort, W. C. Heisenberg exchange enhancement by orbital relaxation in cuprate compounds. Chem. Phys. Lett. 1996, 257, 207-212.

(74) Stoyanova, A.; Sousa, C.; de Graaf, C.; Broer, R. Hopping matrix elements from first principles studies on overlapping fragments: double exchange parameters in manganites. Int. J. Quantum Chem. 2006, 106, 2444-2457.

(75) Huron, B.; Malrieu, J.-P.; Rancurel, P. Iterative perturbation calculations of ground and excited state energies from multiconfigura- 
tional zeroth-order wavefunctions. J. Chem. Phys. 1973, 58, 5745-

5759. 